\title{
Some Inequalities for the Generalized Trigonometric and Hyperbolic Functions
}

\author{
Li Yin ${ }^{1, *}$, Li-Guo Huang ${ }^{1}$, and Feng $\mathbf{Q i}^{2,3,4}$ \\ ${ }^{1}$ Department of Mathematics, Binzhou University, Binzhou City, Shandong Province, China \\ ${ }^{2}$ College of Mathematics, Inner Mongolia University for Nationalities, Tongliao City, Inner Mongolia Autonomous Region, China \\ ${ }^{3}$ Department of Mathematics, College of Science, Tianjin Polytechnic University, Tianjin City, China \\ ${ }^{4}$ Institute of Mathematics, Henan Polytechnic University, Jiaozuo City, Henan Province, China \\ *Corresponding author: yinli_79@163.com
}

Received June 01, 2014; Revised July 01, 2014; Accepted July 10, 2014

\begin{abstract}
In the paper, the authors establish some inequalities of the generalized trigono-metric and hyperbolic functions, partially solve a conjecture posed by R. Klén, M. Vuorinen, and X.-H. Zhang, and finally pose an open problem.
\end{abstract}

Keywords: inequality, generalized trigonometric function, generalized hyperbolic functions, Bernoulli inequality, Huygens' inequality, Wilker's inequality, conjecture, open problem

Cite This Article: Li Yin, Li-Guo Huang, and Feng Qi, "Some Inequalities for the Generalized Trigonometric and Hyperbolic Functions." Turkish Journal of Analysis and Number Theory, vol. 2, no. 3 (2014): 96-101. doi: 10.12691/tjant-2-3-8.

\section{Introduction}

It is well known from calculus that

$$
\arcsin x=\int_{0}^{x} \frac{1}{\left(1-t^{2}\right)^{1 / 2}} d t
$$

and

$$
\frac{\pi}{2}=\arcsin 1=\int_{0}^{1} \frac{1}{\left(1-t^{2}\right)^{1 / 2}} d t
$$

for $0 \leq x \leq 1$. For $1<p<\infty$ and $0 \leq x \leq 1$, the arc sine may be generalized as

$$
\arcsin _{p} x=\int_{0}^{x} \frac{1}{\left(1-t^{p}\right)^{1 / p}} d t
$$

and

$$
\begin{aligned}
& \frac{\pi_{p}}{2}=\arcsin _{p} 1=\int_{0}^{1} \frac{1}{\left(1-t^{p}\right)^{1 / p}} d t=\frac{\pi}{p} \csc \frac{\pi}{p} \\
& =\frac{1}{p} \Gamma\left(\frac{1}{p}\right) \Gamma\left(1-\frac{1}{p}\right)=\frac{1}{p} \int_{0}^{\infty} \frac{t^{1 / p-1}}{1+t} d t,
\end{aligned}
$$

where $\Gamma$ denotes the classical gamma function. Hence, we have $\pi_{p}=\frac{2 \pi}{p \sin (\pi / p)}$.

The inverse of $\arcsin _{p} x$ on $\left[0, \frac{\pi_{p}}{2}\right]$ is called the generalized sine function, denoted by $\sin _{p} x$, and may be extended to $(-\infty, \infty)$. See [7] and closely related references therein.

For $x \in\left[0, \frac{\pi_{p}}{2}\right]$, the generalized cosine function $\cos _{p} x$ is defined by

$$
\cos _{p} x=\frac{d \sin _{p} x}{d x} .
$$

It is easy to see that

$$
\cos _{p} x=\left(1-\sin _{p}^{p} x\right)^{1 / p}
$$

and

$$
\frac{d \cos _{p} x}{d x}=-\cos _{p}^{2-p} x \sin _{p}^{p-1} x
$$

The generalized tangent function $\tan _{p} x$ is defined as

$$
\tan _{p} x=\frac{\sin _{p} x}{\cos _{p} x}, x \in \mathbb{R} \backslash\left\{k \pi_{p}+\frac{\pi_{p}}{2}: k \in \mathbb{Z}\right\} .
$$

From (1.8), it follows that

$$
\frac{d \tan _{p} x}{d x}=1+\left|\tan _{p} x\right|^{p}, x \in\left(-\frac{\pi_{p}}{2}, \frac{\pi_{p}}{2}\right) .
$$

The generalized secant function $\sec _{p} x$ is defined as

$$
\sec _{p} x=\frac{1}{\cos _{p} x}, x \in\left[0, \frac{\pi_{p}}{2}\right) .
$$


It follows from (1.8) and (1.9) that

$$
\sec _{p}^{p} x=1+\tan _{p}^{p} x, x \in\left(0, \frac{\pi_{p}}{2}\right)
$$

and

$$
\frac{d \sec _{p} x}{d x}=\sec _{p} x \tan _{p}^{p-1} x, x \in\left[0, \frac{\pi_{p}}{2}\right) .
$$

The generalized cosecant function $\csc _{p} x$ may be defined as

$$
\csc _{p} x=\frac{1}{\sin _{p} x}, x \in\left(0, \frac{\pi_{p}}{2}\right] .
$$

It is clear that

$$
\csc _{p} x=1+\frac{1}{\tan _{p}^{p} x} \text { and } \frac{d \csc _{p} x}{d x}=-\frac{\csc _{p} x}{\tan _{p} x}(1.14)
$$

for $x \in\left(0, \frac{\pi_{p}}{2}\right)$.

The generalized inverse hyperbolic sine function $\arcsin h_{p} x$ is defined by

$$
\arcsin h_{p} x= \begin{cases}\int_{0}^{x} \frac{1}{\left(1+t^{p}\right)^{1 / p}} d t, & x \in[0, \infty), \\ -\arcsin h_{p}(-x), & x \in(-\infty, 0) .\end{cases}
$$

The inverse of $\arcsin h_{p} x$ is called the generalized hyperbolic sine function and denoted by $\sin h_{p} x$.

The generalized hyperbolic cosine function $\cos h_{p} x$ is defined as

$$
\cos h_{p} x=\frac{d \sin h_{p} x}{d x} .
$$

It is easy to show that

$$
\cos h_{p} x-\left|\sin h_{p} x\right|^{p}=1, x \in \mathbb{R}
$$

and

$$
\frac{d \cos h_{p} x}{d x}=\cos h_{p}^{2-p} x \sin h_{p}^{p-1} x, x \geq 0 .
$$

The generalized hyperbolic tangent function and the generalized hyperbolic secant function are defined as

$$
\tanh _{p} x=\frac{\sin h_{p} x}{\cos h_{p} x} \text { and } \operatorname{sech}_{p} x=\frac{1}{\cos h_{p} x} .
$$

Their derivatives are

$$
\frac{d \tanh _{p} x}{d x}=1-\tanh _{p}^{p} x=\operatorname{sech}_{p}^{p} x
$$

and

$$
\frac{d \operatorname{sech}_{p} x}{d x}=-\operatorname{sech}_{p} x \tanh _{p}^{p-1} x
$$

The above formulas (1.5) to (1.9) and (1.15) to (1.19) can be found in [8]. For $r>0$, the set $S_{2}=\left\{(x, y) \in \mathbb{R}^{2}: x^{2}-y^{2}=r^{2}\right\} \quad$ is the equilateral hyperbola in the plane $\mathbb{R}^{2}$ with the $\ell_{2}$ metric. The connection between the hyperbolic coordinates $(r, \phi)$ and the rectangular coordinates $(x, y)$ is given by $x=r \cosh \phi$ and $y=r \sinh \phi$. We may easily obtain that, when $x, y>0, x^{2}-y^{2}=r^{2}$ and $\phi$ are related by $\phi=\operatorname{arctanh}\left(\frac{y}{x}\right)$. When $p \neq 2$, the analogue of the equilateral hyperbola is the p-equilateral hyperbola

$$
S_{p}=\left\{(x, y) \in \mathbb{R}^{p}: x^{p}-y^{p}=r^{p}\right\}
$$

and the identities $x=r \cosh _{p} \phi$ and $y=r \sinh _{p} \phi$ hold. From this, it follows that

$$
\left|\cosh _{p} \phi\right|^{p}-\left|\sinh _{p} \phi\right|=1
$$

and

$$
\cosh _{p} \phi=\left[1+\left(\sinh _{p} \phi\right)^{p}\right]^{1 / p}, x, y>0 .
$$

This gives a geometrical interpretation to $\sinh _{p} x$ and $\cosh _{p} x$. Furthermore, we may also define the generalized trigonometric functions by means of Gauss hypergeometric function. Interested readers may refer to [1].

As well as we known, the \hyperbolic" function were introduced in 1760 independent by Vincenzo Riccati and John Heinrid Lambert, the notations sh and ch are still used in some other languages such as European, French, and Russian. The hyperbolic function occurs in the solutions of some linear differential equations, such as defining catenary, Laplaces equations in Cartesian coordinates, and occurs in many important areas in physics, such as special relativity. In complex analysis, the hyperbolic function arises the imaginary parts of sine and cosine. For complex variables, the hyperbolic functions are rational functions of exponential functions and are meromorphic. Therefore, many advantages properties relating to the hyperbolic function have already been applied extensively. For more information on this topic, please read the classical book [5].

During the last decades, many authors have studied the generalized trigonometric functions introduced in [9]. See, for example, $[1,2,3,4,7]$ and plenty of references therein. In [8], some classical inequalities for generalized trigonometric and hyperbolic functions, such as Mitrinović-Adamović inequality, Huygens' inequality, and Wilker's inequality were generalized. In [6], some basic properties of the generalized $(p, q)$ - trigonometric functions were given. Recently, the functions $\arcsin _{p, q} x$ and $\operatorname{arcsinh}_{p, q} x$ were expressed in terms of Gaussian hypergeometric functions and many properties and inequalities for generalized trigonometric and hyperbolic functions were established in [3]. In [1], some Turán type inequalities for generalized trigonometric and hyperbolic 
functions were presented. Very recently, a conjecture posed in [3] was verified in [7].

In this paper, we will establish some inequalities of the generalized trigonometric and hyperbolic functions, partially solve a conjecture in [8], and finally pose an open problem.

\section{Lemmas}

For proving our main results, we need the following lemmas.

Lemma 2.1 ([[12], Lemma 2.9]). Let $f$ and $g$ be continuous on $[a, b]$ and differentiable on $(a, b)$ such that $g^{\prime}(x) \neq 0$ on $(a, b)$. If $\frac{f^{\prime}(x)}{g^{\prime}(x)}$ is increasing (or decreasing, respectively) on $(a, b)$, so are the functions $\frac{f(x)-f(b)}{g(x)-g(b)}$ and $\frac{f(x)-f(a)}{g(x)-g(a)}$.

Lemma 2.2 ([[10], Bernoulli inequality]). For $t>-1$ and $\alpha>1$, we have

$$
(1+t)^{\alpha}>1+\alpha t
$$

Lemma 2.3 ([8, Theorem 3.4]). For $p \in[2, \infty)$ and $x \in\left(0, \frac{\pi_{p}}{2}\right)$, we have

$$
\frac{\sin _{p} x}{x}<\frac{x}{\sinh _{p} x} .
$$

Lemma 2.4 ([[8], Theorem 3.16]). If $p>1$, then

$$
\frac{p \sin _{p} x}{x}+\frac{\tan _{p} x}{x}>1+p, 0<x<\frac{\pi_{p}}{2}
$$

and

$$
\frac{p \sinh _{p} x}{x}+\frac{\tanh _{p} x}{x}>1+p, x>0 .
$$

Lemma 2.5 ([[8], Theorem 3.22]). For $p \in(1,2]$, the double inequality

$$
\frac{\sin _{p} x}{x}<\frac{\cos _{p} x+p}{1+p} \leq \frac{\cos _{p} x+2}{3}
$$

holds for all $x \in\left(0, \frac{\pi_{p}}{2}\right]$.

Lemma 2.6 ([[8], Theorem 3.24]). For all $x>0$,

1. if $p \in(1,2]$, then

$$
\frac{\sinh _{p} x}{x}<\frac{\cosh _{p} x+p}{1+p}
$$

2. if $p \in(2, \infty)$, then

$$
\frac{\sinh _{p} x}{x}<\frac{\cosh _{p} x+2}{3} .
$$

Lemma 2.7 ([8, Theorems 3.6 and 3.7]). For all $p \in(1, \infty)$, we have

$$
\cos _{p}^{\alpha} x<\frac{\sin _{p} x}{x}<1, x \in\left(0, \frac{\pi_{p}}{2}\right)
$$

and

$$
\cosh _{p}^{\alpha} x<\frac{\sinh _{p} x}{x}<\cosh _{p}^{\beta} x, x>0
$$

where the constants $\alpha=\frac{1}{p+1}$ and $\beta=1$ are the best possible.

Lemma 2.8. For $p>1$ and $x \in\left(0, \frac{\pi_{p}}{2}\right)$, the function $f(x)=\frac{\sinh _{p} x}{\sin _{p} x}$ is positive and strictly increasing.

Proof. It is apparent that the function $f(x)$ is positive. An easy computation yields $f^{\prime}(x)=\frac{g(x)}{\sin _{p}^{2} x}$ and

$$
g^{\prime}(x)=\left(\tanh _{p}^{p-2} x+\tan _{p}^{p-2} x\right) \sin _{p} x \sinh _{p} x>0,
$$

where

$$
g(x)=\cosh _{p} x \sin _{p} x-\sinh _{p} x \cos _{p} x .
$$

This means that $g(x)$ is strictly increasing and $g(x)>g(0)=0$. Hence, it follows that $f^{\prime}(x)>0$ and that $f(x)$ is strictly increasing.

Lemma 2.9. For $p>1$ and $x \in\left(0, \frac{\pi_{p}}{2}\right)$, the functions $g_{1}(x)=\sin _{p} x-x \cos _{p} x$ and $g_{2}(x)=x \cosh _{p} x-\sinh _{p} x$ are positive.

Proof. An easy computation yields

$$
g_{1}^{\prime}(x)=x \cos _{p} x \tan _{p}^{p-1} x>0
$$

and

$$
g_{2}^{\prime}(x)=x \cosh _{p} x \tanh _{p}^{p-1} x>0 .
$$

These imply that $g_{1}(x)>g_{1}(0)=0$ and that $g_{2}(x)>g_{2}(0)=0$.

\section{Main Results}

Now we are in a position to present our main results.

Theorem 3.1. For $p>1$ and $x \in\left(0, \frac{\pi_{p}}{2}\right)$ we have

$$
\left(\frac{\sin _{p} x}{x}\right)^{p}+\frac{\tan _{p} x}{x}>2
$$

Proof. Setting $t=\frac{\sin _{p} x}{x}-1 \in(-1,0)$ and $\alpha=p$ in (2.1) leads to 


$$
\begin{aligned}
\left(\frac{\sin _{p} x}{x}\right)^{p} & >1+p\left(\frac{\sin _{p} x}{x}-1\right) \\
& >1-p+1+p-\frac{\tan _{p} x}{x}=2-\frac{\tan _{p} x}{x} .
\end{aligned}
$$

Further using (2.3) results in the inequality (3.1).

Remark 3.1. The inequality (3.1) is an analogy of Wilker's inequality involving the sine and tangent functions. See [[12], Section 8.1].

Theorem 3.2. For $p \in[2, \infty)$ and $x \in\left(0, \frac{\pi_{p}}{2}\right)$, we have

$$
\left(\frac{x}{\sinh _{p} x}\right)^{p}+\frac{\tan _{p} x}{x}>2
$$

Proof. Letting $t=\frac{x}{\sinh _{p} x}-1$ and $\alpha=p$ in (2.1) gives

$$
\begin{aligned}
\left(\frac{x}{\sinh _{p} x}\right)^{p} \geq 1 & -p+p \frac{x}{\sinh _{p} x} \\
& >1-p+p \frac{\sin _{p} x}{x}>2-\frac{\tan _{p} x}{x}
\end{aligned}
$$

Combining this with (2.2) and (2.3) yields (3.2).

Theorem 3.3. For $p \in(1,2]$ and $x \in\left(0, \frac{\pi_{p}}{2}\right)$ we have

$$
\frac{p x}{\sin _{p} x}+\frac{x}{\tan _{p} x}>1+p .
$$

Proof. This follows from using (2.5) and

$$
\begin{gathered}
p+\cos _{p} x-(1+p) \frac{\sin _{p} x}{x} \\
>p+\cos _{p} x-(1+p) \frac{\cos _{p} x+p}{1+p}=0 .
\end{gathered}
$$

Theorem 3.4. For $p \in(1,2]$ and $x>0$, we have

$$
\frac{p x}{\sinh _{p} x}+\frac{x}{\tanh _{p} x}>1+p \text {. }
$$

Proof. This follows from using (2.6) and

$$
\begin{gathered}
p+\cosh _{p} x-(1+p) \frac{\sinh _{p} x}{x} \\
>p+\cosh _{p} x-(1+p) \frac{\cosh _{p} x+p}{1+p}=0 .
\end{gathered}
$$

Remark 3.2. Inequalities presented in Theorems 3.3 and 3.4 are analogies of Huygens' inequality for the sine and tangent functions. See [11].

Theorem 3.5. For $p \in(1,2]$, we have

$$
\left(\frac{x}{\sin _{p} x}\right)^{p}+\frac{x}{\tan _{p} x}>2, \quad x \in\left(0, \frac{\pi_{p}}{2}\right)
$$

and

$$
\left(\frac{x}{\sinh _{p} x}\right)^{p}+\frac{x}{\tanh _{p} x}>2, \quad x>0
$$

Proof. Taking $t=\frac{x}{\sin _{p} x}-1$ and $\alpha=p$ in (2.1) and using the inequality (3.3) result in

$$
\begin{aligned}
\left(\frac{x}{\sin _{p} x}\right)^{p} & >1+p\left(\frac{x}{\sin _{p} x}-1\right) \\
& >1-p+1+p-\frac{x}{\tan _{p} x}=2-\frac{x}{\tan _{p} x} .
\end{aligned}
$$

The inequality (3.6) may be deduced similarly.

Theorem 3.6. For $p \in(1, \infty)$ and $x \in\left(0, \frac{\pi_{p}}{2}\right)$ we have

$$
\left(\frac{\sin _{p} x}{x}\right)^{p}+\frac{\tan _{p} x}{x}>\left(\frac{x}{\sin _{p} x}\right)^{p}+\frac{x}{\tan _{p} x}
$$

Proof. When letting $a=\left(\frac{\sin _{p} x}{x}\right)^{p}$ and $b=\frac{\tan _{p} x}{x}$ the inequality (3.7) becomes $a+b>\frac{1}{a}+\frac{1}{b} \quad$ which is equivalent to $a b>1$, that is,

$$
\left(\frac{\sin _{p} x}{x}\right)^{p+1} \frac{1}{\cos _{p} x}>1
$$

This can be derived from utilizing (2.8) as follows

$$
\left(\frac{\sin _{p} x}{x}\right)^{p+1} \frac{1}{\cos _{p} x}>\left[\cos _{p}^{1 /(p+1)} x\right]^{p+1} \frac{1}{\cos _{p} x}=1 .
$$

Theorem 3.7. For $p \in(1, \infty)$ and $x>0$, we have

$$
\left(\frac{\sinh _{p} x}{x}\right)^{p}+\frac{\tanh _{p} x}{x}>\left(\frac{x}{\sinh _{p} x}\right)^{p}+\frac{x}{\tanh _{p} x} .
$$

Proof. This follows from using the inequality (2.9).

Remark 3.3. Inequalities (3.7) and (3.8) imply inequalities (3.1) and (3.20) in [8, Corollary 3.19].

Theorem 3.8. For $p>1$ and $x \in\left(0, \frac{\pi_{p}}{2}\right)$, we have

$$
\frac{\tan _{p} x}{x}>\frac{x}{\sin _{p} x}
$$

Proof. Let $f(x)=\tan _{p} x \sin _{p} x-x^{2}$. An easy computation yields

$$
\begin{aligned}
f^{\prime}(x)= & \sin _{p} x \sec _{p}^{p} x+\sin _{p} x-2 x, \\
f^{\prime \prime}(x)= & \cos _{p} x \sec _{p}^{p} x+p \sin _{p} x \sec _{p}^{p} x \tan _{p}^{p-1} x \\
& +\cos _{p} x-2, \\
f^{\prime \prime \prime}(x)= & {\left[(2 p-1) \sec _{p}^{p} x-1\right] \sin _{p}^{p-1} x \sec _{p}^{p-2} x } \\
& +p^{2} \sin _{p}^{2 p-1} x \sec _{p}^{3 p-2} x \\
& +p(p-1) \sin _{p}^{p-1} x_{p}^{3 p-2} x \\
\geq & 0 .
\end{aligned}
$$


Hence, $f^{\prime \prime}(x)>f^{\prime \prime}(0)=0, f^{\prime}(x)$ is strictly increasing, $f^{\prime}(x)>f^{\prime}(0)=0$, and $f(x)>f(0)=0$. The inequality (3.9) follows.

Theorem 3.9. For $p>1$ and $x \in(0, \infty)$, we have

$$
\frac{\tanh _{p} x}{x}>\frac{p+1}{p \cosh _{p} x+1}
$$

or, equivalently,

$$
\frac{\sinh _{p} x}{x}>\frac{(p+1) \cosh _{p} x}{p \cosh _{p} x+1} .
$$

Proof. Set $f(x)=(p+1) x-\tanh _{p} x\left(p \cosh _{p} x+1\right)$. Then

$$
f^{\prime}(x)=p-p \cosh _{p} x+\tanh _{p}^{p} x
$$

and

$$
f^{\prime \prime}(x)=p \tanh _{p}^{p-1} x\left(\sec _{p}^{p} x-\cosh _{p} x\right)<0 .
$$

Accordingly, it follows that $f^{\prime}(x)<f^{\prime}(0)=0, f^{\prime}(x)$ is strictly decreasing, and $f(x)<f(0)=0$. The inequalities (3.10) and (3.11) are proved.

Remark 3.4. Considering Theorem 3.9 and $\cosh _{p} x>1$ reveals

$$
\frac{\sinh _{p} x}{x}>\frac{p+1}{p+\cosh _{p} x}
$$

Theorem 3.10. For $p \geq 3$ and $x \in\left(x_{p}^{*}, \frac{\pi_{p}}{2}\right)$, the function $f(x)=\frac{\ln \frac{x}{\sin _{p} x}}{\ln \frac{\sinh x}{x}}$ is strictly increasing, where $x_{p}^{*}$ satisfies the equation $\cosh _{p}^{p} x=p-2$.

Proof. Let $f_{1}(x)=\ln \frac{x}{\sin _{p} x}$ and $f_{2}(x)=\ln \frac{\sinh _{p} x}{x}$. Then $f_{1}(0)=\lim _{x \rightarrow 0} f_{1}(x)=0, \quad f_{2}(0)=\lim _{x \rightarrow 0} f_{2}(x)=0$, and

$$
\frac{f_{1}^{\prime}(x)}{f_{2}^{\prime}(x)}=\frac{\sinh _{p} x}{\sin _{p} x} \frac{g_{1}(x)}{g_{2}(x)}
$$

where $\quad g_{1}(x)=\sin _{p} x-x \cos _{p} x \quad$ and $g_{2}(x)=x \cosh _{p} x-\sinh _{p} x \quad$ satisfy $\quad g_{1}(0)=g_{2}(0)=0$ and

$$
\begin{aligned}
& \frac{g_{1}^{\prime}(x)}{g_{2}^{\prime}(x)}=\frac{\sin _{p} x \tan _{p}^{p-2} x}{\sinh _{p} x \tanh _{p}^{p-2} x}, \\
& \frac{d}{d x}\left[\frac{g_{1}^{\prime}(x)}{g_{2}^{\prime}(x)}\right]=\frac{\tan _{p}^{p-3} x \tanh _{p}^{p-3} x h(x)}{\left(\sinh _{p} x \tanh _{p}^{p-2} x\right)^{2}}, \\
& h(x)=\left[(p-2) \sec _{p}^{p} x-1\right] \tan _{p} x \\
& \quad+\left[1-(p-2) \operatorname{sech}_{p}^{p} x\right] \tan _{p} x .
\end{aligned}
$$

Owing to $p \geq 3$ and $x \in\left(x_{p}^{*}, \frac{\pi_{p}}{2}\right)$, by the monotonicity of $\sec _{p} x$ and $\operatorname{sech}_{p} x$, we obtain $(p-2) \sec _{p}^{p} x-1>0$ and $1-(p-2) \operatorname{sech}_{p}^{p} x>0$. So the ratio $\frac{g_{1}^{\prime}(x)}{g_{2}^{\prime}(x)}$ is strictly increasing for $x \in\left(x_{p}^{*}, \frac{\pi_{p}}{2}\right)$. By Lemma 2.1, we see that $\frac{g_{1}(x)}{g_{2}(x)}$ is strictly increasing for $x \in\left(x_{p}^{*}, \frac{\pi_{p}}{2}\right)$. On account of Lemmas 2.8 and 2.9, we obtain that the quotient $\frac{f_{1}^{\prime}(x)}{f_{2}^{\prime}(x)}$ is strictly increasing. Thus, by Lemma 2.1, the ratio $\frac{f_{1}(x)}{f_{2}(x)}$ is also strictly increasing.

Remark 3.5. If $p=3$, we obtain $x_{3}^{*}=0$. This means that the function $f(x)=\frac{\ln \frac{x}{\sin _{3} x}}{\ln \frac{\sinh _{3} x}{x}}$ is strictly increasing on $\left(0, \frac{\pi_{3}}{2}\right)$

Notice that Theorem 3.10 partially solves Conjecture 3.12 in [8].

Theorem 3.11. For $p>1$ and $x \in(0,1)$, we have

$$
\int_{0}^{1} \frac{\cos _{p} x}{\sqrt{1-x^{p}}} d x>\int_{0}^{1} \frac{x^{p-2} \sin _{p} x}{p \sqrt{1-x^{p}}} d x
$$

Proof. If putting $t=\arcsin _{p} x$, the left hand side of (3.14) becomes

$$
\int_{0}^{1} \frac{\cos _{p} x}{p \sqrt{1-x^{p}}} d x=\int_{0}^{\pi p / 2} \cos _{p} \sin _{p} t d t .
$$

Similarly, taking $t=\arccos _{p} x$, the right hand side of (3.14) reduces to

$$
\int_{0}^{1} \frac{x^{p-2} \sin _{p} x}{p \sqrt{1-x^{p}}} d x=\int_{0}^{\pi_{p} / 2} \sin _{p} \cos _{p} t d t .
$$

Making use of the monotonicity of $\sin _{p} x$ and $\cos _{p} x$ acquires

$$
\cos _{p} \sin _{p} t>\cos _{p} t>\sin _{p} \cos _{p} t .
$$

The inequality (3.14) is thus proved.

\section{An Open Problem}

Finally, we pose an open problem.

$$
\frac{p \sin _{p} x}{x}+\frac{\tan _{p} x}{x}>\frac{p x}{\sin _{p} x}+\frac{x}{\tan _{p} x}
$$


is valid on $\left(0, \frac{\pi_{p}}{2}\right)$.

\section{Acknowledgements}

The first author was supported partially by the NSF of Shandong Province under grant numbers ZR2011AL001, ZR2012AQ028, and by the Science Foundation of Binzhou University under grant BZXYL1303, China.

The authors appreciate the anonymous referees for their valuable comments on and helpful corrections to the original version of this paper.

\section{References}

[1] Á. Baricz, B. A. Bhayo, and M. Vuorinen, Turán type inequalities for generalized inverse trigonometric functions, available online at http://arxiv.org/abs/1305.0938.

[2] B. A. Bhayo and M. Vuorinen, Inequalities for eigen-functions of the p-Laplacian, available online at http: //arxiv.org/abs/1101.3911.
[3] B. A. Bhayo and M. Vuorinen, On generalized trigonometric functions with two parameters, J. Approx. Theory 164 (2012), no. $10,1415-1426$.

[4] B. A. Bhayo and M. Vuorinen, Power mean inequality of generalized trigonometric functions, available online at http://arxiv.org/abs/1209.0873.

[5] B. C. Carlson, Special Functions of Applied Mathematics Academic Press [Harcourt Brace Jovanovich, Publishers], New York-London, 1977.

[6] D. E. Edmundes, P. Gurka, and J. Lang, Properties of generalized trigonometric functions, J. Approx. Theory 164 (2012), no. 1, 4756.

[7] W.-D. Jiang, M.-K. Wang, Y.-M. Chu, Y.-P. Jiang, and F. Qi, Convexity of the generalized sine function and the generalized hyperbolic sine function, J. Approx. Theory 174 (2013), 1-9.

[8] R. Klén, M. Vuorinen, and X.-H. Zhang, Inequali ties for the generalized trigonometric and hyperbolic functions, J. Math. Anal. Appl. 409 (2014), no. 1, 521-529.

[9] P. Lindqvist, Some remarkable sine and cosine functions, Ricerche Mat. 44 (1995), no. 2, 269-290 (1996).

[10] D. S. Mitrinovic, Analytic inequalities, Springer, New York, 1970.

[11] E. Neumann and J. Sándor, On some inequalities involving trigonometric and hyperbolic functions with emphasis on the Cusa-Huygens, Wilker, and Huygens inequalities, Math. Inequal. Appl. 13 (2010), no. 4, 715-723.

[12] F. Qi, D.-W. Niu, and B.-N. Guo, Refinements, generalizations, and applications of Jordan's inequality and related problems, $\mathrm{J}$. Inequal. Appl. 2009 (2009), Article ID 271923, 52 pages. 